\title{
A Parametric LQ Approach to Multiobjective Control System Design
}

\author{
Doug Ky \\ Control Systems Branch \\ NASA Lewis Research Center \\ Cleveland, Ohio 44135
}

\section{Abstract}

In this paper the synthesis of a constant parameter output feedback control law of constrained structure is set in a multiple objective linear quadratic regulator (MOLQR) framework. The use of intuitive objective functions such as model-following ability and closed-loop trajectory senfunctions such as model-following ability and closed-loop trajectory sen-
sitivity, allow multiple objective decision making techniques, such as the surrogate worth trade-off method, to be applied. For the continuous-time deterministic problem with an infinite time horizon, dynamic compensators as well as static output feedback controllers can be synthesized using a descent Anderson-Moore algorithm that is modified to impose linear equality constraints on the feedback gains by moving in feasible directions. Results of three different examples are presented, including a unique reformulation of the sensitivity reduction problem.

Introduction

In synthesizing a control law a designer has two objectives in mindmaximize performance and minimize the cost of implementation. A simpler control law, which is less complicated and less costly to implement than, for example, a full state feedback controller, may be preferred by the designer. The designer may have a number of structural alternatives in mind such as full output feedback, decentralized control, or low order dynamic compensation. In this paper the problem of minimizing the cost of implementation is viewed as a problem of selecting a control law among a number of alternative control law structures. The trade-offs between the performance objective and the cost of implementation can only be examined fairly if the free parameters of each structure optimize the performance objective. The performance objective itself may be characterized as a set of objectives. The objectives considered in this paper are integral quadratic objectives of state energy, control energy, model following ability, trajectory sensitivity, and subsystem objectives. These objectives are often conflicting, therefore a multiple objective approach can be taken.

Algorithms for constant parameter output feedback controllers have been extensively studied [1]-[17]. Few algorithms however, allow the designer to arbitrarily prespecify the control law structure [6], [7], [11], [14], [17]. Fewer still consider the multiple objective nature of the problem [13][15]. Three functional scalarization methods have been applied to MOLQR problems - the weighted sum, $\epsilon$-constraint, and goal attainment methods. All three methods convert the multiple objective problem into a single objective problem. A single noninferior solution may then be found by solving the single objective problem using any one of a number of nonlinear programming techniques or algorithms that solve the necessary conditions.

The weighted sum method is used in this paper. The solution algorithm used is a descent Anderson-Moore algorithm modified to move in feasible directions. This algorithm was chosen for modification because it has been shown by example to be faster than the Davidon-Fletcher-Powell method [8], and because proofs of convergence to a local stationary point of the cost function of Anderson-Moore type algorithms have been reported on [8], [11] (these proofs are contested however in [15].)

This paper is organized into two parts. In part one, the constrained optimal output feedback problem is formulated and a solution algorithm is presented. In part two, multiple objective LQR problems are formulated and results of examples are summarized.

\subsection{Optimal Controllers of Constrained Structure}

\subsection{Full output feedback controllers}

The linear dynamic multiple-input multiple-output plant model is in the standard state space form

$$
\begin{aligned}
& \dot{x}(t)=A x(t)+B u(t) \\
& y(t)=C x(t)
\end{aligned}
$$

where $x \in R^{n}$ is the state vector, $u \in R^{m}$ is the input vector, and $y \in R^{p}$ is the output vector. The outputs are assumed to be the sensor measurements. The initial state, or initial condition, is given by $x(0)=x_{0}$.

U.S. Government Work not Protected by U.S. Copyright
Marc Buchner

Systems Engineering Dept.

Case Western Reserve University Cleveland, Ohio 44106

$-\sqrt{-1}$

The control law is defined as

$$
u(t)=-G y(t)
$$

where $G \in R^{m \times p}$.

The objective function to be minimized that will be considered here is defined as

$$
J\left(G, x_{0}\right)=\int_{0}^{\infty}\left[x^{T}(t) Q x(t)+u^{T}(t) R u(t)\right] d t
$$

where $Q$ is a positive semi-definite $n \times n$ matrix, $R$ is a positive definite $m \times m$ matrix, and both are symmetric. In order for $J\left(G, x_{0}\right)$ to be finite, the states weighted by the matrix $Q$ must be rendered asymptotically stable by application of a finite amount of control energy.

For any feedback gain matrix $G$ that stabilizes the closed loop system $\widehat{A}=A-B G C$ the value of the objective function may be evaluated as

$$
\begin{aligned}
J\left(G, x_{0}\right) & =x_{0}^{T} K x_{0} \\
& =\operatorname{tr}\left\{K x_{0} x_{0}^{T}\right\}
\end{aligned}
$$

where $K$ is the unique positive semi-definite solution of the linear matrix equation

$$
\widehat{A}^{T} K+K \widehat{A}+Q+(G C)^{T} R G C=0 .
$$

Thus what may have appeared to be a dynamic optimization problem (eqs. (1-1)-(1-3)), is now formulated as a static objective function (1-4) that is to be minimized with respect to $G$ and $K$ subject to the equality constraints (1-5). Finally, the problem may be restated as: Given the dynamic system (1-1), initial condition $x_{0}$, and a control law of the form $u(t)=-G y(t)$, choose $G^{*}$ to minimize the objective function $J\left(G, x_{0}\right)$.

The first-order necessary conditions are found by first forming the Lagrangian

$\mathcal{L}(G, K, L)=\operatorname{tr}\left\{K x_{0} x_{0}^{T}\right\}+\operatorname{tr}\left\{\left[\widehat{A}^{T} K+K \widehat{A}+Q+(G C)^{T} R G C\right] L^{T}\right\}(1-6)$

where $L \in R^{n \times n}$ is a matrix of Lagrange multipliers. By using gradient matrix operations [4], the first-order necessary conditions for $G^{*}, K^{*}$, and $L^{*}$ to be optimal are given by

$$
\begin{aligned}
& \left.\frac{\partial \mathcal{L}}{\partial G}\right|_{*}=2\left[R G^{*} C L^{*} C^{T}-B^{T} K^{*} L^{*} C^{T}\right]=0 \\
& \left.\frac{\partial \mathcal{L}}{\partial K}\right|_{*}=\widehat{A}^{*} L^{*}+L^{*} \widehat{A}^{* T}+x_{0} x_{0}^{T}=0 \\
& \left.\frac{\partial \mathcal{L}}{\partial L}\right|_{*}=\widehat{A}^{* T} K^{*}+K^{*} \widehat{A}^{*}+Q+\left(G^{*} C\right)^{T} R G^{*} C=0
\end{aligned}
$$

where $\widehat{A}^{*}=A-B G^{*} C$. Equation (1-7) may be rewritten as

$$
G^{*}=R^{-1} B^{T} K^{*} L^{*} C^{T}\left(C L^{*} C^{T}\right)^{-1} .
$$

The optimal cost is $J^{*}\left(G^{*}, x_{0}\right)=\operatorname{tr}\left\{K^{*} x_{0} x_{0}^{T}\right\}$.

\subsection{Output feedback controllers of constrained structure}

A full output feedback formulation assumes all the elements of $G$ are free to be optimized. Although full output feedback uses only those measurements that are available, it does not allow the designer freedom to choose feedback paths. Feedback paths may be eliminated by constraining elements of $G$ to be zero. In addition, if the set of available measurements is too restrictive, dynamic compensation may be necessary to stabilize the system. Even if a constrained control law stabilizes the system, the addition of a dynamic compensator may improve performance. Dynamic compensators may be designed by augmenting the state and output vectors and constraining appropriate elements of the output feedback matrix to be zero or one. 
The only difference between the first-order necessary conditions for optimality for a full output feedback controller (1-7)-(1-9) and an output feedback controller of constrained structure (elements of $G$ constrained to be constant) is that only the partials of $\mathcal{L}$ with respect to the free parameters of $G$ need to equal zero. The additional necessary condition $G^{*} \in \Omega$ must, of course, be satisfied.

In order to constrain the structure of an output feedback controller, elements of the feedback gain matrix $G$ are constrained to be constant. These constraints are a special form of linear equality constraints. Linear equality constraints are useful for other purposes as well as constraining the structure of the gain matrix. A robust control law for multiple plants can be designed using a multiple objective approach by constraining corresponding elements of the feedback matrix to be equal (section 2.4.3). Trade-ofis between closed-loop trajectory sensitivity and other LQR objectives can also be examined by constraining elements of the feedback matrix to be equal to each other (section 2.4.5). The initial values of some elements of the control vector may be specified by realizing that the initial control vector is $u(0)=-G C x(0),[14]$. Calise and Raman use linear equality constraints to impose a degree of modal insensitivity on the closed loop system [16].

The optimization problem for the constrained optimal output feedback problem can be stated as: Given the dynamic system (1-1), initial condition $x_{0}$, a control law of the form $u(t)=-G y(t)$, and the constraint set

$$
\Omega=\left\{G \mid h_{i}(G)-b_{i}=0, \quad i=1, \ldots, m\right\}
$$

where the scalar function $h_{i}(G)$ is a linear combination of the elements of $G$, choose $G^{*} \in \Omega$ to minimize the objective function $J\left(G, x_{0}\right)$.

\subsection{Solution Technique}

To enforce the linear equality constraints (1-12) the following penalty function is defined

$$
\gamma(G)=\frac{v}{2} \sum_{i=1}^{m}\left(h_{i}(G)-b_{i}\right)^{2}
$$

The objective function to be minimized becomes $\widehat{J}\left(G, x_{0}\right)=J\left(G, x_{0}\right)+$ $\gamma(G)$. The Moerder-Calise algorithm [11] may be applied directly to this problem formulation. However with the penalty function method the solution is never feasible and the stationary points of $\widehat{J}$ are not the stationary points of $J$ unless $\gamma(G)=0$. The constraints must be forced to be satisfied, and then the resulting feasible solution evaluated. Increasing the weighting factor $v$ to a large value may cause problems with convergence of the algorithm.

In order to avoid any problems associated with penalty functions, a descent Anderson-Moore algorithm is modified to move in feasible directions only. A feasible direction is obtained by projecting the unconstrained direction onto the linear equality constraints at each iteration of the algorithm. Define the vector $\chi$ as the vector of elements of the matrix $G$. The linear equality constraints (1-12) can also be represented by the matrix equation

$$
A_{c} \chi=b \text {. }
$$

If $d_{k}$ is the unconstrained direction at step $k$ of the algorithm, then $\hat{d}_{k}$ the projected feasible direction is [24]

$$
\hat{d}_{k}=-\left[I-A_{\varepsilon}^{T}\left(A_{\varepsilon} A_{\varepsilon}^{T}\right)^{-1} A_{c}\right] d_{k} .
$$

Define $\Delta G$ to be the unconstrained direction and $\Delta \widehat{G}$ to be the projected direction. In this paper the following two constraints and associated projections are used:

1) To constrain the gain element $g_{i j}$ equal to a constant $c_{i j}$, the projection

$$
\Delta \widehat{g}_{i j}=0 .
$$

2) To constrain $g_{i j}$ equal to $g_{k l}$, the projection is

$$
\Delta \widehat{g}_{i j}=\Delta \widehat{g}_{k l}=\left(\frac{\Delta g_{i j}+\Delta g_{k l}}{2}\right) .
$$

Two rules were used to change the sign of the elements of $\Delta \widehat{G}$ in order to ensure an improvement in cost at each step of the algorithm:

1) For $\widehat{g}_{i j}$ unconstrained, change the sign of $\Delta \widehat{g}_{i j}$ to ensure that

$$
\frac{\partial J}{\partial g_{i j}} \Delta \widehat{g}_{i j} \leq 0 \text {. }
$$

2) For the constraint $\Delta \widehat{g}_{i j}=\Delta \widehat{g}_{k l}$, change the sign of both $\Delta \widehat{g}_{i j}$ and $\Delta \widehat{g}_{k l}$ to ensure that

$$
\frac{\partial J}{\partial g_{i j}} \Delta \widehat{g}_{i j}+\frac{\partial J}{\partial g_{k l}} \Delta \widehat{g}_{k l} \leq 0 \text {. }
$$

To a first order approximation, this results in a reduction in cost along each vector of a basis formed by the unconstrained gains $\Delta \widehat{g}_{i j}$ and the constraints $\widehat{\Delta} g_{i j}=\widehat{\Delta} g_{k l}$. Note that, in general, these type of 'sign change' rules only apply when the linear equality constraints $h_{i}(G), i=1, \ldots, m$ are independent.

1.3.1 The Algorithm

The modified descent Anderson-Moore algorithm for computing $G^{*}$ is:

$0)$ Choose $G_{0} \in \Omega$ such that $\widehat{A}=A-B G_{0} C$ is stable. Set $i=0$.

1) Solve (1-9) for $K_{i}$ and (1-8) for $L_{i}$.

2) Evaluate

$$
\Delta G_{i}=R^{-1} B^{T} K_{i} L_{i} C^{T}\left(C L_{i} C^{T}\right)^{-1}-G_{i}
$$

3) Use equations (1-16) and (1-17) to project $\Delta G_{i}$ onto the constraint set $\Omega$ and obtain $\Delta \hat{G}_{i}$.

4) Use rules (1-18) and (1-19) to change signs of elements of $\Delta \hat{G}_{i}$ if necessary, where

$$
\frac{\partial J}{\partial G}=2\left[R G_{i} C L_{i} C^{T}-B^{T} K_{i} L_{i} C^{T}\right]
$$

5) Set

$$
G_{i+1}=G_{i}+\alpha \Delta \widehat{G}_{i}
$$

where $\alpha \in[0,1)$ is chosen to ensure

$$
J_{i+1}<J_{i}=\operatorname{tr}\left\{K_{i} x_{0} x_{0}^{T}\right\} .
$$

6) Set $i=i+1$, go to 1$)$.

Practical methods for determining an initial stabilizing gain $G_{0}$ may be found in [9]. The algorithm was programmed in Turbo-Pascal on an IBM PC-XT. Step 1 requires the solution of two Lyapunov equations (1-8) and (1-9). See Smith [25], or the more efficient algorithm of Bartels-Stewart [26]. A method for selecting $\alpha$ is needed in step 5. The convergence proof of Moerder and Calise [11] shows that there exists an $\alpha$ small enough so that $J_{i+1}<J_{i}$. Therefore the simplest method is to start with $\alpha=1$ then reduce $\alpha$ if necessary to ensure a reduction in cost. The algorithm, as described, lacks a stopping criteria, however the criteria $\left|\partial J / \partial G_{i}\right|<\epsilon_{1}$ or $J_{i}-J_{i+1}<\epsilon_{2}$ may be used to stop the algorithm.

\subsection{Multiobjective LQR Problems}

\subsection{Problem statement}

The problem is to optimize simultaneously $N$ objective functions. The multiple objective problem, or vector optimization problem, is stated as:

$$
\min _{G \in \Omega}\left\{J_{1}(G), J_{2}(G), \ldots, J_{N}(G)\right\}
$$

where $G$ is a vector (or matrix) of decision variable or optimizing parameter values and $\Omega$ is a constraint set. Each objective function $J_{i}(G)$ is an integral quadratic objective function similar to $J\left(G, x_{0}\right)$. The explicit reference to the initial condition $x_{0}$ is omitted for notational convenience.

If the objective functions are conflicting, which is often the case, the global optimums of $J_{i}(G), i=1, \ldots, N$, are not achieved at the same $G^{*}$. Clearly there is no unique solution to the problem. However, a set $\Omega^{*}$ of noninferior solutions (or Pareto optimal solutions), can be defined.

DEFINITION: A decision $G^{*}$ is said to be a noninferior solution to the problem posed by $(2-1)$, if there does not exist another $\bar{G}$ so that $J_{j}(\bar{G}) \leq J_{j}\left(G^{*}\right), j=1,2, \ldots, N$, with strict equality holding for at least one $j$ [18]

Therefore a noninferior solution is one in which a further optimization of any $J_{i}(G)$ is at the expense of at least one of the others. (i.e., all $J_{i}(G)$ 's cannot be reduced simultaneously). The concept of a noninferior surface is graphically illustrated by the two objective, single decision variable problem in figure 1 where objective functions $J_{1}(g)$ and $J_{2}(g)$ are plotted versus the single decision variable $g$. The objective functions $J_{1}(g)$ and $J_{2}(g)$ achieve 
their optimums at $g_{1}^{*}$ and $g_{2}^{*}$ respectively. The values of $g$ between $g_{1}^{*}$ and $g_{2}^{*}$ are noninferior solutions to the problem because a further decrease in either objective leads to an increase in the other. The noninferior surface (or trade-off surface) is plotted in the decision space in figure 2.

Thus the problem of finding a solution that is acceptable to the contro system designer is reduced to finding an acceptable solution in the noninferior set, $\Omega^{*}$, rather than the entire feasible set, $\Omega$. To solve the overall problem then, a technique for generating noninferior solutions is needed as well as a technique for determining which noninferior solution the decision maker (control systems designer) prefers.

2.2 Generating noninferior solutions

In this paper the weighted sum method is used. Noninferior solutions are generated by solving a single objective problem that is the weighted sum of the $N$ objective functions. The problem is stated as:

$$
\min _{G \in \Omega} \sum_{i=1}^{N} w_{i} J_{i}(G), \quad \sum_{i=1}^{N} w_{i}=1, \quad 0 \leq w_{i} \leq 1
$$

Geometrically, for a two objective problem, the solution of (2-2) is represented by the closest line to the origin, of slope $-w_{2} / w_{1}$, that is tangent to the noninferior surface (figure 2). For any set of weights satisfying $\sum_{i} w_{i}=1$ the solution to $(2-2)$ is a noninferior solution. However, the weighted sum method fails to find all points on a nonconvex trade-of surface. In figure 3 the noninferior solutions between points $A$ and $B$ cannot be found using the weighted sum method. The weighted sum method has the advantage that if the weighted sum of objective functions maintains the same form as a single objective function, an algorithm that applies to the single objective problem may be directly applied to the multiple objective problem. This will be shown to be the case for MOLQR problems.

\subsection{Deciding on an acceptable noninferior solution}

Although a nonlinear programming algorithm may be used to find a single noninferior solution, the problem of finding a noninferior solution that is preferred by the decision maker (the control systems designer) must that is preferred by the decision maker (the control systems designer) must
still be addressed. In making a decision it is typically assumed the decision maker maximizes a utility function i.e.

$$
\max _{G \in \Omega^{*}} U\left[J_{1}(G), \ldots, J_{N}(G)\right]
$$

where $U[\cdot]$ is a monotonic decreasing function of the objective functions $J_{i}(G)$, and $\Omega^{*}$ is the set of all noninferior solutions. There are many interactive techniques that can be used for extracting from the decision maker the additional information necessary to solve (2-5) [19]. A technique which requires a minimum amount of information is the surrogate worth trade-off (SWT) method [18], [19]. The SWT method uses the values of the trade-off functions, defined as

$$
\lambda_{i j}=-\left.\frac{\partial J_{i}}{\partial J_{j}}\right|_{G \in \Omega^{*}}
$$

in questioning the decision maker. The decision maker (DM) is asked a trade-off question such as: "Given levels of objectives $J_{1}(G), \ldots, J_{N}(G)$ how willing are you to trade $\lambda_{i j}$ units of $J_{i}$ to reduce $J_{j}$ by one unit?" The $\mathrm{DM}$ is questioned about a sequence of noninferior points until a point is found such that the decision maker is indifferent to moving in any direction.

All iterative methods assume the decision maker can judge the worth of the objectives $J_{i}(G)$. For an MOLQR formulation to be valid the designer must have enough understanding of each single objective in order to answer the trade-off question effectively.

\subsection{MOLQR formulations and examples}

The purpose of this section is to formulate some useful multiple objec tive linear quadratic regulator (MOLQR) problems in which the designe can address the trade-off question.

2.4.1 The general linear quadratic regulator problem

The integral quadratic objective function

$$
J\left(G, x_{0}\right)=\int_{0}^{\infty}\left[x^{T}(t) Q x(t)+u^{T}(t) R u(t)\right] d t
$$

is a sum of terms

$$
\begin{array}{ll} 
\pm q_{i j} \int_{0}^{\infty} x_{i}(t) x_{j}(t) d t & i, j=1, \ldots, n \\
\pm r_{k i} \int_{0}^{\infty} u_{k}(t) u_{l}(t) d t & k, l=1, \ldots, m
\end{array}
$$

with the weights $q_{i j} \geq 0$ and $r_{k l}>0$ chosen to ensure $Q \geq 0, R>0$, and both $Q$ and $R$ symmetric. The weights may be normalized so that $\sum_{i, j} q_{i j}+\sum_{i, j} r_{i j}=1$. Thus the solution to (2-7) may be interpreted as a noninferior solution to the multiple objective problem consisting of the objectives weighted in (2-8) and (2-9). To arrive at a noninferior solution that is acceptable to the designer the weights are changed and the problem is solved again. However the objectives in (2-8) and (2-9) containing cross terms (e.g., $\left.x_{i}(t) x_{j}(t), i \neq j\right)$ in general have no physical meaning to the designer. Thus it is difficult, if not impossible, for the designer to answer the question: "Are you willing to trade $\lambda_{i j}$ units of $J_{i}$ for one unit of $J_{j}$ ?" The problem may be reformulated as follows: Consider objective functions of the form

$\int_{0}^{\infty} x_{i}^{2}(t) d t, \quad \int_{0}^{\infty}\left(x_{i}(t)-x_{j}(t)\right)^{2} d t, \quad \int_{0}^{\infty}\left(x_{i}(t)+x_{j}(t)\right)^{2} d t$

$\int_{0}^{\infty} u_{i}^{2}(t) d t, \quad \int_{0}^{\infty}\left(u_{i}(t)-u_{j}(t)\right)^{2} d t, \quad \int_{0}^{\infty}\left(u_{i}(t)+u_{j}(t)\right)^{2} d t$

A standard LQR objective function (2-7) is obtained by weighting these six terms such that $Q \geq 0, R>0$, and both $Q$ and $R$ are symmetric. The integrands of the integral equations in (2-10) and (2-11) are positive for all $t$, therefore weighting the objectives in (2-10) by $\hat{g}_{i j}>0$ ensures that $Q \geq 0$ and weighting the objectives in (2-11) by weights $\hat{r}_{i j}>0$ ensures that $R>0$. This observation motivates the following theorem.

\section{THEOREM 2-1:}

Given a multiple objective linear quadratic regulator (MOLQR) problem that is defined as a weighted sum of objective functions as

$$
\begin{aligned}
J(G) & =\int_{0}^{\infty}\left[x^{T}(t) Q x(t)+u^{T}(t) R u(t)\right] d t \\
& =\sum_{i=1}^{N} w_{i} J_{i},
\end{aligned}
$$

the noninferior surface between the $N$ objectives $J_{i}$ is convex for a full state feedback control law if for every set of weights that satisfies

1) $0 \leq w_{i} \leq 1, i=1, \ldots, N$

2) $\sum_{i=1}^{N} w_{i}=1$

the penalty matrices $Q$ and $R$ satisfy

3) $Q$ positive semi-definite $(Q \geq 0)$, and symmetric

4) $R$ positive definite $(R>0)$ and symmetric.

PROOF:

The proof is by contradiction. Assume that the noninferior surface is nonconvex. Then there must exist a set of weights such that there are two (or more) different $G^{*}$ that minimize $J(G)$. By assumption, for every set of weights satisfying 1 ) and 2$), Q \geq 0$ and $R>0$, therefore the minimum of $J(G)$ is unique. Thus there does not exist a set of weights satisfying 1 ) and 2) which results in two (or more) different $G^{*}$ minimizing $J(G)$. The noninferior surface therefore must be convex.

The objectives in (2-10) and (2-11) have more meaning to the designer, in that they are easier to trade-off, than those of (2-8) and (2-9).

\subsubsection{Integrated control system design}

Integrated control system design deals with the integrated design of subsystem controllers. The overall system is viewed as consisting of $l$ subsystems with the system state and control vectors $x(t)$ and $u(t)$ separated into subsystem state vectors $x_{i}(t)$ and control vectors $u_{i}(t), i=1, \ldots, l$ Each subsystem has a different objective function

$$
J_{i}(G)=\int_{0}^{\infty}\left[x_{i}^{T}(t) Q_{i} x_{i}(t)+u_{i}^{T}(t) R_{i} u_{i}(t)\right] d t .
$$

To solve this problem using the weighted sum method the single objective

$$
J(G)=\sum_{i=1}^{l} w_{i} J_{i}(G), \quad \sum_{i=1}^{l} w_{i}=1, \quad 0 \leq w_{i} \leq 1
$$

which is of the form (2-7), is minimized subject to any constraints on the control law. The weighting matrices $Q$ and $R$ take on the form

$$
\begin{aligned}
& Q=\text { block } \operatorname{diag}\left\{w_{1} Q_{1}, w_{2} Q_{2}, \ldots, w_{l} Q_{l}\right\} \\
& R=\text { block } \operatorname{diag}\left\{w_{1} R_{1}, w_{2} R_{2}, \ldots, w_{l} R_{l}\right\} .
\end{aligned}
$$


Fennel and Black compute a single noninferior point for an integrated airframe and propulsion control problem using the weighted sum method [23]. The weights were chosen to be the inverse of the optimums of the single objective problems, i.e., $w_{i}=1 / J_{i}^{*}$ where $J_{i}^{*}$ is the optimum for the single objective problem of minimizing $J_{i}$. The objective function was optimized for a full state feedback structure by solving a Riccati equation. Suboptimal solutions of different structures, including a decentralized tion. Suboptimal solutions of different structures, including a decentralized structure, were obtained by zeroing elements of the optimum full state feed-
back gain matrix. Caglayan, Halyo, and Broussard reformulate Fennel and Black's integrated airframe and propulsion control problem as a discrete time stochastic problem [10]. The weighted sum of objective functions is optimized subject to a decentralized control law using an iterative algorithm developed in [10]. However, neither of the above references ([10] [23]) investigate the trade-offs between airframe and engine performance, or the trade-offs between controllers of different structures. This was done or the trade-ofs between controllers of different structures. This was done
in [27] using the modified algorithm. The trade-offs are summarized in figure 4. Significant cost reduction was obtained using the modified algorithm compared to the suboptimal techniques in [23], table 1.

\subsubsection{Multiple regime control system design}

In a multiple regime control system design problem it is assumed that the plant may be any plant in a finite set of $l$ time-invariant plants. The problem is to find the best control law that simultaneously optimizes the performance objectives of each plant in the set. Each plant has a different performance objectiv
objective function

$$
J_{i}(G)=\int_{0}^{\infty}\left[x_{i}^{T}(t) Q_{i} x_{i}(t)+u_{i}^{T}(t) R_{i} u_{i}(t)\right] d t
$$

where $x_{i}(t)$ and $u_{i}(t)$ are the state and control vector for the $i^{\text {th }}$ plant. To solve this problem using the weighted sum method, vectors $x(t), y(t)$, and $u(t)$ are defined which consist of the vectors $x_{i}(t), y_{i}(t)$, and $u_{i}(t)$, of the $l$ plants. The scalar objective function $J(G)$ then satisfies $(2-13)$ and $(2-7)$ with weighting matrices

$$
\begin{aligned}
& Q=\text { block } \operatorname{diag}\left\{w_{1} Q_{1}, w_{2} Q_{2}, \ldots, w_{l} Q_{l}\right\} \\
& R=\text { block } \operatorname{diag}\left\{w_{1} R_{1}, w_{2} R_{2}, \ldots, w_{l} R_{l}\right\} .
\end{aligned}
$$

In order to find the single best control law for all the plants in the set, the control laws of the plants must be constrained to be equal to each other by the constraint

$$
\begin{aligned}
& G=\text { block } \operatorname{diag}\left\{G_{1}, G_{2}, \ldots, G_{l}\right\} \\
& G_{1}=G_{2}=\cdots=G_{l} .
\end{aligned}
$$

The plants in the set could represent different operating conditions or failure modes. The expected cost $\mathrm{E}\{J\}=\sum_{i} p_{i} J_{i}(G)$ could then be minimized, where $p_{i}$ is the probability of occurrence of plant $i$. In terms of decision theory, the problem is now one of decision making under uncertainty. The expected cost should be modified to include a weighting term $w_{i}$ for each term $p_{i} J_{i}(G)$. The term $w_{i}$ is determined based on the decision makers trade-offs. For example the decision maker may be unwilling to take the risk of incurring a large cost $J_{k}(G)$ even if there is a small probability of occurrence of plant $k$.

Fleming and Pashkevich use the goal attainment method to design a controller for a robot arm that operates in three different regimes [13]. The weighting coefficients $w_{i}=J_{i}^{*}$, where $J_{i}^{*}$ is the single objective minimum were chosen so that the same degree of under-attainment is achieved in each objective. In [27] a proportional plus integral controller is tuned for two possible plants, $G_{1}(s)=(1 / s+1), G_{2}(s)=2 /(4 s+1)$. The objective is to minimize the model following error of the closed loop step input response of both plants, where the model is $G_{m}(s)=36 /\left(s^{2}+7.2 s+36\right)$. A slowly decaying exponential is used to approximate a step input. The trade-of decaying exponential is used to approxinate a step input. The trade-of surface between model-following objectives $J_{1}(G)$ and $J_{2}(G)$ is shown in
figure 5 . The closed loop step responses for both plants for a weighting factor of .5 is shown in figure 6. Greater model-following for plant 2 may be obtained (at the expense of plant 1) by solving the problem for a weight of $<.5$.

\subsubsection{Model-following objectives}

If a model of the desired closed-loop plant is known, the modelfollowing objective

$$
\int_{0}^{\infty}\left(x_{m}(t)-x(t)\right)^{T} Q_{m}\left(x_{m}(t)-x(t)\right) d t
$$

may be used to minimize model-following error where $x_{m}(t)$ is the state vector of the model. The problem can be formulated by defining a state vector which includes both $x(t)$ and $x_{m}(t)$.

The $\epsilon$-constraint method is used by Fleming [14] on two flight control system examples. A longitudinal controller for a helicopter is designed by minimizing a model-following term subject to control energy constraints on each of the helicopter's two inputs. In the second example a control system for an aerodynamically unstable aircraft is designed by minimizing sensitivity subject to constraints on model-following errors and control energy. The trade-offs between model-following and sensitivity are examined.

2.4.5 Closed-loop trajectory sensitivity as an objective

Consider systems of the form

$$
\begin{aligned}
\dot{x}(t) & =A(p) x(t)+B(p) u(t) \\
y(t) & =C x(t)
\end{aligned}
$$

where $x(t), u(t)$, and $y(t)$ are defined earlier (1-1) and $p \in R^{r}$ is a vector of parameters. To simplify notation only the single parameter case will be discussed below although the results easily extend to the multiple parameter case. The closed-loop trajectory sensitivity due to changes in a parameter $p_{1}$ may be reduced by adding the term

$$
\int_{0}^{\infty}{\frac{\partial x^{T}}{\partial p_{1}}}^{T} Q_{1} \frac{\partial x}{\partial p_{1}} d t
$$

to the objective function (2-7) [20], [22]. The closed-loop trajectory sensitivity function $S_{1}(t)=\partial x(t) / \partial p_{1}$ is described by the state equations

$$
\begin{aligned}
& \dot{S}_{1}(t)=A(p) S_{1}(t)+\frac{\partial A(p)}{\partial p_{1}} x(t)+\frac{\partial B(p)}{\partial p_{1}} u(t)+B(p) \frac{\partial u(t)}{\partial p_{1}} \\
& S_{1}(0)=0 .
\end{aligned}
$$

The term $\partial u(t) / \partial p_{1}$ depends on the form of the control law. Fleming and Newmann [20] augment the state vector $x(t)$ with the sensitivity vector $S_{1}(t)$. A full state feedback controller is then

$$
u(t)=-F_{1} x(t)-F_{2} S_{1}(t) .
$$

To implement this control law $S_{1}(t)$ must be simulated. However when the control law $(2-22)$ is substituted into $(2-21)$ a term $\partial S_{1}(t) / \partial p_{1}$ appears. Fleming and Newmann neglect this term. Hafez and Loparo [22] derive the necessary conditions for optimality for the control law

$$
u(t)=-F x(t) \text {. }
$$

Unfortunately the algorithm of section 1.3 .1 cannot be directly applied to the necessary condition in [22] because the matrix $Q$ is a function of the feedback gain matrix $F$.

However, the problem can be put into a form in which the algorithm can be applied directly. This is done by augmenting the control vector $u(t)$ with $\partial u(t) / \partial p_{1}$ and constraining the control law so that (2-21) holds. Given the control law (2-23) the formula for $\partial u(t) / \partial p_{1}$ is

$$
\frac{\partial u(t)}{\partial p_{1}}=-F S_{1}(t)
$$

Substituting (2-24) into (2-21) and evaluating at $p=p^{0}$ the state equations describing $S_{1}(t)$ become

$$
\dot{S}_{\mathrm{1}}(t)=A S_{1}(t)+A_{1} x(t)-B_{1} u(t)-B u_{S_{1}}(t)
$$

where the input $u_{S_{1}}(t)=\partial u(t) / \partial p_{1}=-F S_{1}(t)$ has been defined and the following notation has been used

$$
A=A\left(p^{0}\right), \quad A_{1}=\left.\frac{\partial A(p)}{\partial p_{1}}\right|_{p^{0}} \quad B=B\left(p^{0}\right), \quad B_{1}=\left.\frac{\partial B(p)}{\partial p_{1}}\right|_{p^{0}}
$$

The problem is then formulated as an optimal output feedback problem with a constrained control law as follows:

$$
\begin{aligned}
\left(\begin{array}{c}
\dot{x}(t) \\
\dot{S_{1}}(t)
\end{array}\right)= & \left(\begin{array}{cc}
A & 0 \\
A_{1} & A
\end{array}\right)\left(\begin{array}{c}
x(t) \\
S_{1}(t)
\end{array}\right)+\left(\begin{array}{cc}
B & 0 \\
B_{1} & B
\end{array}\right)\left(\begin{array}{c}
u(t) \\
u_{S_{1}}(t)
\end{array}\right) \\
y(t)= & I\left(\begin{array}{c}
x(t) \\
S_{1}(t)
\end{array}\right) \\
\left(\begin{array}{c}
u(t) \\
u_{S_{1}}(t)
\end{array}\right)= & -\left(\begin{array}{cc}
F & 0 \\
0 & F
\end{array}\right)\left(\begin{array}{c}
x(t) \\
S_{1}(t)
\end{array}\right) \\
& \hat{Q}=\left(\begin{array}{cc}
Q & 0 \\
0 & Q_{1}
\end{array}\right) \quad \widehat{R}=\left(\begin{array}{cc}
R & 0 \\
0 & \epsilon I
\end{array}\right)
\end{aligned}
$$

The closed-loop trajectory sensitivity may be further reduced by including a dynamic compensator with state vector $z(t)$ described by

$$
\left(\begin{array}{c}
u(t) \\
\dot{z}(t)
\end{array}\right)=\left(\begin{array}{cc}
E & H \\
B_{z} & A_{z}
\end{array}\right)\left(\begin{array}{c}
x(t) \\
z(t)
\end{array}\right)
$$


therefore

$$
\frac{\partial u(t)}{\partial p_{1}}=E \frac{\partial x(t)}{\partial p_{1}}+H \frac{\partial z(t)}{\partial p_{1}} .
$$

The state vector must be further extended by the compensator state trajectory sensitivity function $S_{z_{1}}(t)=\partial z(t) / \partial p_{1}$, leading to the following state equations

$$
\dot{S}_{z_{1}}(t)=A_{z} S_{z_{1}}(t)+B_{z} \frac{\partial x(t)}{\partial p_{1}} .
$$

The state equation for $S_{1}(t)$ is found by defining the input

$$
u_{S_{1}}(t)=\frac{\partial u(t)}{\partial p_{1}}=E S_{1}(t)+H S_{z_{1}}(t) .
$$

The reformulated problem for optimizing (2-7) plus (2-20) subject to the control law (2-29) is:

$$
\begin{aligned}
& \left(\begin{array}{c}
\dot{x} \\
\dot{z} \\
\dot{S}_{1} \\
\dot{S}_{z_{1}}
\end{array}\right)=\left(\begin{array}{cccc}
A & 0 & 0 & 0 \\
0 & 0 & 0 & 0 \\
A_{1} & A & 0 & 0 \\
0 & 0 & 0 & 0
\end{array}\right)\left(\begin{array}{c}
x \\
z \\
S_{1} \\
S_{z_{1}}
\end{array}\right)+\left(\begin{array}{cccc}
B & 0 & 0 & 0 \\
0 & I & 0 & 0 \\
B_{1} & 0 & B & 0 \\
0 & 0 & 0 & I
\end{array}\right)\left(\begin{array}{c}
u \\
\dot{z} \\
u_{S_{1}} \\
\dot{S}_{z_{1}}
\end{array}\right) \\
& \left(\begin{array}{c}
u \\
\dot{z} \\
u_{S_{1}} \\
\dot{S}_{z_{1}}
\end{array}\right)=\left(\begin{array}{cccc}
E & H & 0 & 0 \\
B_{z} & A_{z} & 0 & 0 \\
0 & 0 & E & H \\
0 & 0 & B_{z} & A_{z}
\end{array}\right)\left(\begin{array}{c}
x \\
z \\
S_{1} \\
S_{z_{1}}
\end{array}\right) \\
& \widehat{Q}=\text { block } \operatorname{diag}\left\{Q, 0, Q_{1}, 0\right\} \quad \hat{R}=\text { block } \operatorname{diag}\{R, \epsilon I, \epsilon I, \epsilon I,\}
\end{aligned}
$$

It is important to note that in some cases greater insensitivity of the response due to a change in commanded variables might be obtained using open-loop compensation as opposed to feedback control. The closed-loop trajectory sensitivity to additional parameters $p_{i}$ may be considered by extending the state vector with the sensitivity functions $S_{i}(t)$.

The closed-loop trajectory sensitivity to the relative position of the center of gravity of an unstable aircraft is investigated in references [21], [14], and [27]. Results using the modified algorithm [27], for a full state feedback control law, are presented in tables 2 and 3 , and figures 7 and 8 . feedback control law, are presented in tables 2 and 3 , and figures 7 and 8 .
The objective function is $J=(1-w) J_{m}+w J_{s}+J_{u}$, where $J_{m}$ is the model following error, $J_{s}$ is the sensitivity measure, and $J_{u}$ is the control energy. The main trade-off in the reduction of sensitivity is the increase in control energy

\subsection{Summary}

In this paper the synthesis of a linear output feedback control law of a specified structure has been set in a multiple objective framework. The designer may trade-off any number of individual integral quadratic objectives such as state energy, control energy, model-following, trajectory sensitivity, and subsystem objectives. Noninferior solutions may be generated by applying the modified descent Anderson-Moore algorithm of section 2.5 to a single objective problem that is the weighted sum of the objective functions. Dynamic compensators as well as static output feedback controlíers can be designed using linear equality constraints on the parameters of the output feedback matrix to impose the structural constraints.

\section{References}

[1] W. S. Levine and M. Athans, "On the determination of optimal constant output feedback gains for linear multivariable systems," IEEE Trans. Automatic Contr., vol. AC-15, pp. 44-48, Feb. 1970.

[2] T. L. Johnson and M. Athans, "On the design of optimal constrained dynamic compensators for linear constant systems," IEEE Trans. Audynamic compensators for linear constant systems,"

[3] H. P. Horisberger and P. R. Belanger, "Solution of the optimal constant output feedback problem by conjugate gradients," IEEE Trans. Automatic Contr., vol. AC-19, pp. 434-435, Aug. 1974

[4] J. M. Mendel, "A concise derivation of optimal constant limited state feedback gains," IEEE Trans. Automatic Contr., pp. 447 448, Aug. 1974.

[5] T. Söderström, "On some algorithms for design of optimal constrained regulators," IEEE Trans. Automatic Contr., vol. AC-23, pp. 11001101, Dec. 1978 .

[6] C. Wenk and C. Knapp, "Parameter optimization in linear systems with arbitrarily constrained controller structure" IEEE Trans. Automatic Contr., vol. AC-25, pp. 496-500, June 1980.

[7] Y. G. Srinivasa and T. Rajogopalan, "Algorithms for the computation of optimal output feedback gains" "Proc. 18th IEEE Conf Decision Contr., Fort Lauderdale, FL, Dec. 1979.

[8] N. Halyo and J. R. Broussard, "A convergent algorithm for the stochastic infinite-time discrete optimal output feedback problem," Proc. 1981 JACC, Charlottesville, VA,1981.
[9] J. R. Broussard and N. Haylo, "Active flutter control using discrete optimal constrained dynamic compensators" Proc. Amer. Contr. Conf. San Diego, CA, pp. 1026-1034, June 1983.

[10] A. Caglayan, N. Halyo, and J. Broussard, "The use of the optimal output feedback algorithm in integrated control system design" Nat. Aerosp. Electron. Conf., Dayton, OH, May 1983.

[11] D. D. Moerder and A. J. Calise, "Convergence of a numerical algoithm for calculating optimal output feedback gains," IEEE Trans. Automatic Contr., vol. AC-30, pp. 900-903, Sept. 1985.

[12] J. C. Geromel and J. Bernussou, "Parametrical optimization for decentralized regulation of discrete systems," Control and Dynamic Systems, vol. 23, pp.123-137, 1986.

[13] P. J. Fleming and A. P. Pashkevich, "Computer aided control system design using a multiobjective optimization approach," Proc. IEE Control Conf., 1985.

[14] P. Fleming, "A non-linear programming approach to the computer aided design of

[15] H. T. Toivonen, "A multiple objective linear quadratic gaussian control problem," IEEE Trans. Automatic Contr., vol. AC-29 pp. 279-280, March 1984.

[16] A. J. Calise and K. Raman, "Modal insensitivity with optimality," Proc. Amer. Contr. Conf., San Diego, CA, June 1984

[17] P. M. Mäkilä, and H. T. Toivonen, "Computational methods for parametric LQ problems-a survey," IEEE Trans. Automatic Contr., vol. AC-32, no. 8, Aug. 1987.

[18] Y. Haimes, Hierarchical Analyses of Water Resources Systems, McGraw-Hill, 1977

[19] V. Chankong and Y. Haimes, Multiobjective Decision Making: Theory and Methodology, North-Holland, 1983.

[20] P. J. Fleming and M. M. Newmann, "Design algorithm for sensitivity constrained suboptimal regulator," Int. J. Contr., 1977.

[21] G. Grübel and G. Kreisselmeier, "A generalized sensitivity concept for sensitivity reduction in control system design," Proc. Joint Automatic Control Conf., Austin, Texas, pp. 328-332, 1974.

[22] W. Hafez, K. Loparo, M. Hashish, E. Rasmy, "Sensitivity-constrained controller design for multi-parameter systems," Second IASTED Int. controller design for multi-parameter systems," Second IASTED Int.
Conf. on Applied Control and Identification, Los Angeles, CA, Dec., 1986 .

[23] R. E. Fennel and S. B. Black, "Integrated airframe propulsion control," NASA CR-3606, Aug. 1982.

[24] D. G. Luenberger, Introduction to Linear and Nonlinear Programming, Addison-Wesley Publishing Co., 1973.

[25] P. G. Smith, "Numerical solution of the matrix equation $A X+X A^{T}+$ $B=0$," IEEE Trans. Automatic Contr., vol. AC-, pp. 278-279, June 1971

[26] R. H. Bartels and G. W. Stewart, "Solution of the matrix equation $A X+X B=C$, Communications of the ACM, vol. 15, pp. 820-826, Sept. 1972.

[27] D. Kyr, "Multiple objective control system design using constrained optimal output feedback," M. S. Thesis, Dept. of Systems Engr.,

\section{Acknowledgment}

This research was supported in part by NASA grant NAG3-659. 


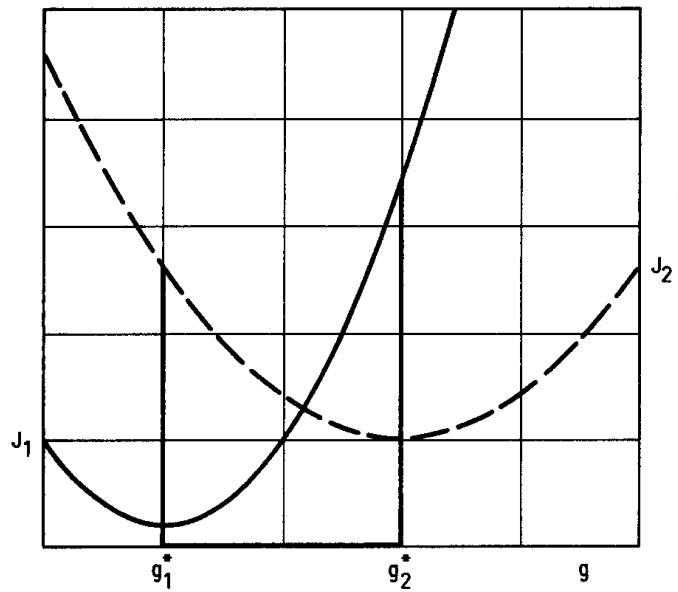

FIGURE 1. - OBJECTIVE FUNCTIONS $J_{1}(g)$ AND $J_{2}(g)$ VER.SUS SINGLE DECISION VARIABLE $g$.

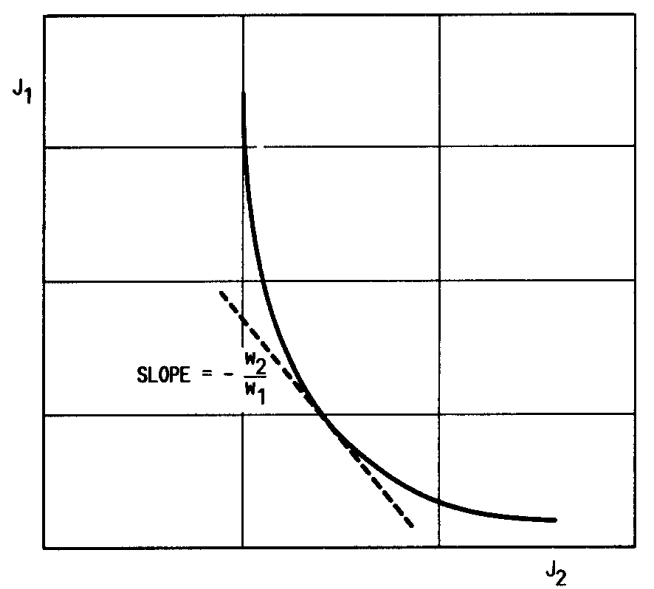

FIGURE 2. - NONIMFERIOR SURFACE BETMEEN THE TWO OBJECTIVES $J_{1}(g)$ AND $J_{2}(g)$ OF FIGURE 1 .

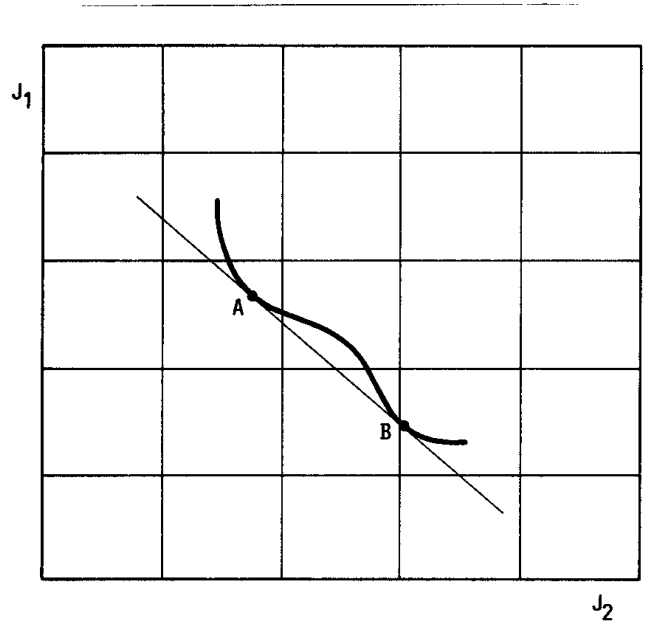

FIGURE 3. - THE WEIGHTED SUN METHOD APPLIED TO A NONCOHVEX TRADE-OFF SURFACE.
TABLE 1. - COMPARISON OF COSTS BETWEEN REFERENCE [23] AND CONSTRAINED OPTIMIZATION, FOR THE INTEGRATED AIRFRAME AND PROPULSION CONTROL EXAMPLE, $\alpha=0.4848$

\begin{tabular}{|c|c|c|c|c|c|c|}
\hline \multirow{2}{*}{$\begin{array}{l}\text { Controller } \\
\text { structure }\end{array}$} & \multicolumn{3}{|c|}{ Reference [23] } & \multicolumn{3}{|c|}{ Constrained optimization } \\
\hline & $\mathrm{J}$ & $\mathrm{J}_{\mathrm{a}}$ & $\mathrm{J}_{\mathrm{e}}$ & $\mathrm{J}^{\star}$ & $\mathrm{J}_{\mathrm{d}}^{*}$ & $J_{e}^{*}$ \\
\hline $\begin{array}{l}\text { Centralized } \\
\text { Decentralized } \\
\text { st } 4(.1)\end{array}$ & $\begin{array}{l}1367 \\
3330 \\
1759\end{array}$ & $\begin{array}{l}29.89 \\
29.40 \\
33.10\end{array}$ & $\begin{array}{l}2624 \\
6422 \\
3329\end{array}$ & $\begin{array}{l}1373 \\
1444\end{array}$ & $\begin{array}{l}\overline{26.95} \\
42.37\end{array}$ & $\begin{array}{l}2641 \\
2763\end{array}$ \\
\hline
\end{tabular}

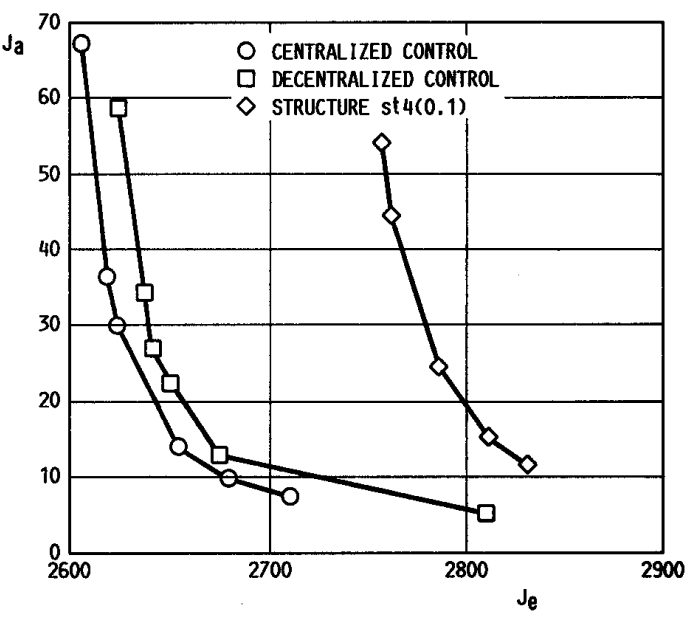

FIGURE 4. - NONINFERIOR SURFACES FOR THE INTEGRATED AIRFRAME AND PROPULSION CONTROL EXAMPLE OF SECTION 2.4.2.

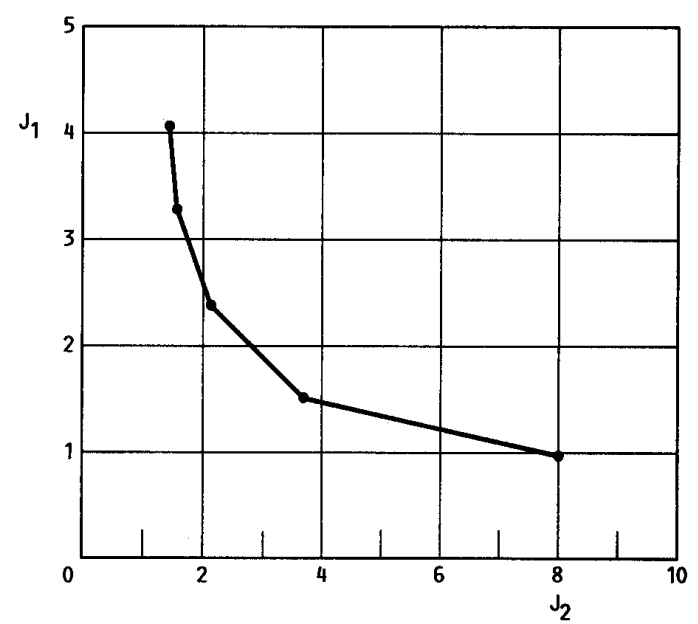

FIGURE 5, - NONINFERIOR SURFACE FOR NONINAL VERSUS OFFMOMINAL PERFORMANCE EXAMPLE OF SECTION 2.4.3. 


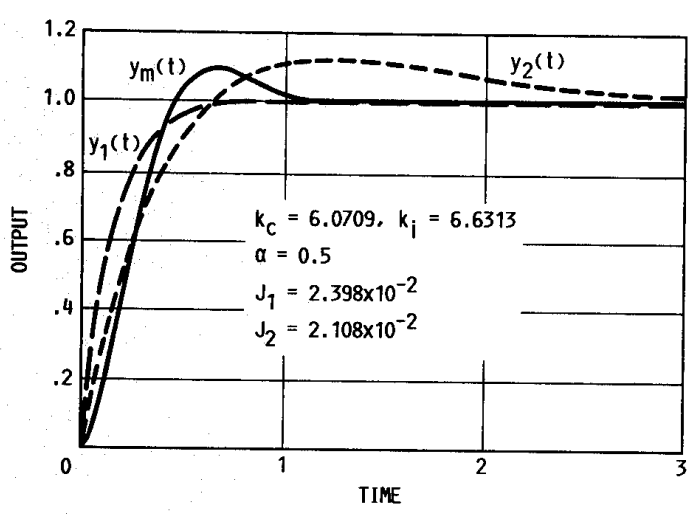

FIGURE 6. - STEP RESPONSES, EXAMPLE 2.4.3.

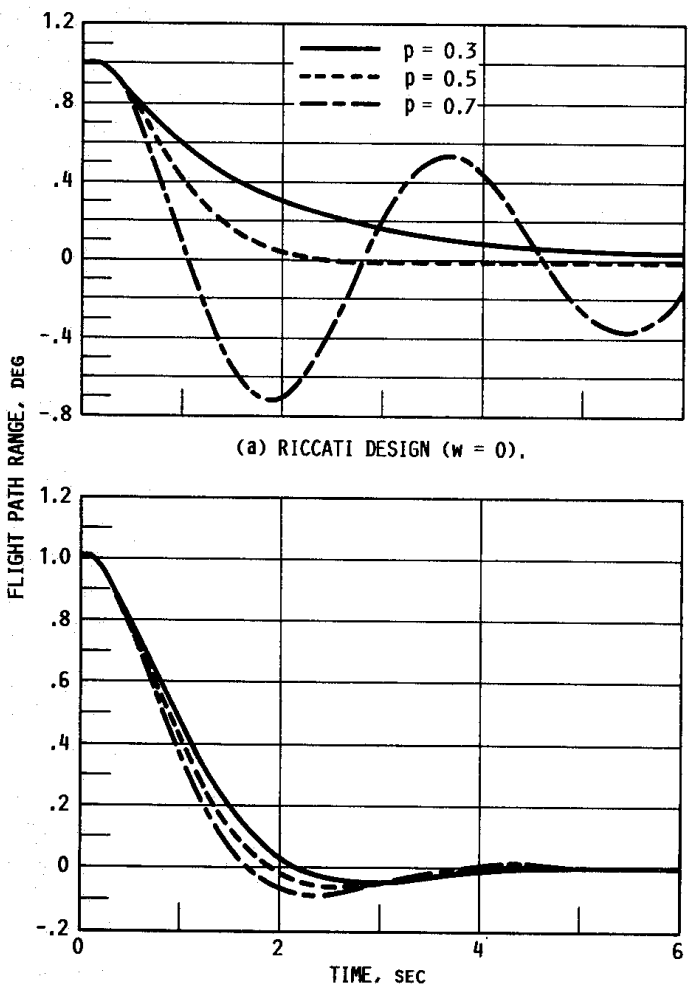

(b) SENSITIVITY REDUCED DESIGN $(W=0.1)$.

FIGURE 7. - TRANSIENT OF FLIGHT PATH ANGLE FOR DIFFERENT PARAMETER VALUES $p$.
TABLE 2. - OPTIMAL COSTS FOR MODEL. FOLLOWING VERSUS SENSITIVITY EXAMPLE OF SECTION 2.4 .5

\begin{tabular}{|c|c|c|c|c|}
\hline$w$ & $\mathrm{~J}_{\mathrm{s}}$ & $\mathrm{J}_{\mathrm{m}}$ & $\mathrm{J}_{\mathrm{u}}$ & $\mathrm{J}$ \\
\hline 0.0 & 5.2697 & $4.1116 \times 10^{-12}$ & $7.767 \times 10^{-2}$ & $4.1116 \times 10^{-12}$ \\
0.1 & .1277 & $1.2278 \times 10^{-01}$ & $2.086 \times 10^{-1}$ & $1.2303 \times 10^{-01}$ \\
\hline
\end{tabular}

TABLE 3. - OPTIMAL GAINS FOR MODEL FOLLOWING VERSUS SENSITIVITY EXAMPLE OF SECTION 2.4.5

\begin{tabular}{|c|c|c|c|c|}
\hline$w$ & \multicolumn{1}{|c|}{$g_{11}^{*}$} & $g_{12}^{*}$ & $g_{13}^{*}$ & $g_{14}^{*}$ \\
\hline 0.0 & -1.4760 & 0.307 & -0.238 & -0.903 \\
0.1 & -.0739 & -3.297 & -3.436 & -2.308 \\
\hline
\end{tabular}

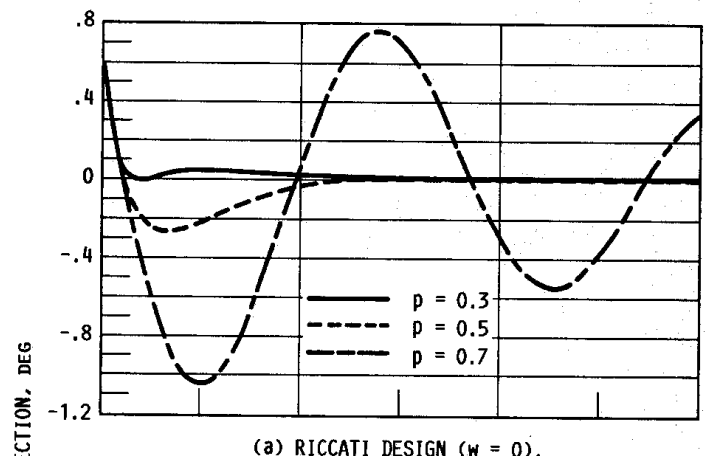

(a) RICCATI DESIGN $(W=0)$.

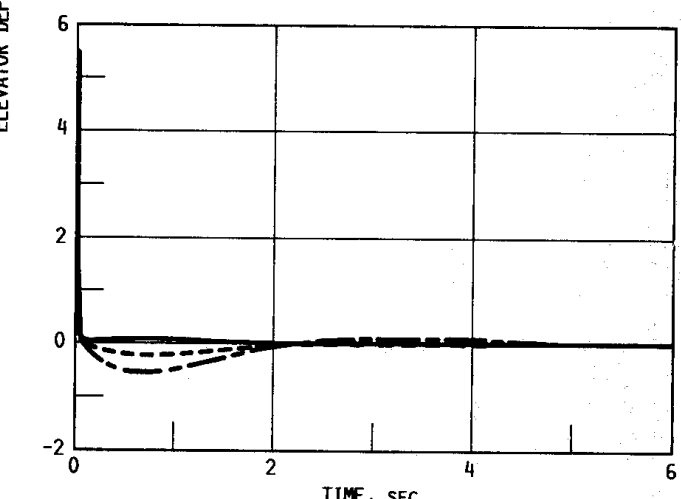

(b) SENSITIVITY REDUCED DESIGN ( $w=0.1)$.

FIGURE 8. - TRANSIENT OF ELEVATOR DEFLECTION FOR DIFFERENT PARAMETER VALUES $p$. 